\title{
Existence of Fixed Points in Fuzzy Strong b-Metric Spaces
}

\author{
Shazia Kanwal $\mathbb{D},{ }^{1}$ Doha Kattan $\mathbb{D D}^{2}$, Saba Perveen, ${ }^{1}$ Sahidul Islam $\mathbb{D D}^{3}$ \\ and Mohammed Shehu Shagari iD ${ }^{4}$ \\ ${ }^{1}$ Department of Mathematics, Government College University Faisalabad, Faisalabad, Pakistan \\ ${ }^{2}$ Department of Mathematics, King Abdulaziz University, Rabigh, Saudi Arabia \\ ${ }^{3}$ Department of Mathematics, Jahangirnagar University Savar, Savar, Dhaka, Bangladesh \\ ${ }^{4}$ Department of Mathematics, Ahmadu Bello University, Zaria, Nigeria
}

Correspondence should be addressed to Shazia Kanwal; shaziakanwal@gcuf.edu.pk and Sahidul Islam; sahidul.sohag@juniv.edu Received 22 November 2021; Accepted 24 December 2021; Published 15 January 2022

Academic Editor: Naeem Jan

Copyright (C) 2022 Shazia Kanwal et al. This is an open access article distributed under the Creative Commons Attribution License, which permits unrestricted use, distribution, and reproduction in any medium, provided the original work is properly cited.

In the present research, modern fuzzy technique is used to generalize some conventional and latest results. The objective of this paper is to construct and prove some fixed-point results in complete fuzzy strong b-metric space. Fuzzy strong b-metric (sbmetric) spaces have very useful properties such as openness of open balls whereas it is not held in general for b-metric and fuzzy b-metric spaces. Due to its properties, we have worked in these spaces. In this way, we have generalized some well-known fixedpoint theorems in fuzzy version. In addition, some interesting examples are constructed to illustrate our results.

\section{Introduction}

In pure mathematics, the theory of fixed points is the most dynamic and active area of research. The theory of fixed points has already been revealed as a great and significant weapon for studying nonlinear analysis. In literature, we observe that many scholars put their efforts in this field of research; for instance, see [1-8] and references therein.

In 1965, Zadeh [9] introduced a very beautiful idea, which is a tool that makes possible the description of vagueness, imprecision, and manipulations with their notions. Fuzzy set theory is very interesting and more beneficial than classic set theory. That is why it gained much attention of researchers and scholars. So, these techniques are applied in diverse fields of engineering, fractals, image processing, navigation, and many other fields of science. For example, fuzzy fixed-point theory [6-8], fuzzy group theory [10], fuzzy ring theory $[11,12]$, fuzzy field [13], and fuzzy differential equations (see reference in these mentioned papers, for more detail).

In 1975, Kramosil and Michalek [14] introduced fuzzy metric space which is a generalization of probabilistic metric space; later on, George and Veeramani [15] introduced the notion of a fuzzy metric space. This work lays a solid foundation for the expansion of fixed-point theory in fuzzy metric space. Then, Grabiec [16] explained the completeness of the fuzzy metric space, and the Banach contraction theorem was extended to complete fuzzy metric spaces. Fang [17] further sets some latest fixed-point theorems for contractive-type mappings in G-complete fuzzy metric space by following Grabiec's work. Along with fuzzy metric spaces, some more extensions of metric and metric space terms are existed.

In 1989, Bakhtin [18] instigates a space in which a weaker condition was observed instead of the triangle inequality, with the goal of generalizing the Banach contraction principle [19] and extensively used by Czerwic [20]. They called these spaces $b$-metric spaces. The topology persuade by a b-metric contains few "unpleasant" functions. For example, open balls may not be open, closed balls may not be closed, and a b-metric may not be continuous as a mapping in the induced topology. In 2019, in the middle of the classes of b-metric spaces and metric spaces, Kirk and Shahzad [21] instigate the class of strong b-metric spaces by using the inequality $d\left(a_{1}, a_{2}\right) \leq d\left(a_{1}, a_{3}\right)+s d\left(a_{3}, a_{2}\right)$ for all $a_{1}, a_{2}, a_{3} \in \Omega$ and $s \geq 1$. 
Strong b-metric space has the advantage over b-metric spaces that, in the induced topology, open balls are open, so they stake a number of characteristics that are the same to those of classic metric space. Recently, in 2019, Oner and Sostak [22] have introduced the definition and properties of strong fuzzy b-metric space. Thus, the class of fuzzy sbmetric spaces lies between fuzzy metric spaces and fuzzy b-metric spaces. As expected, fuzzy sb-metric spaces have useful properties similar to metric and fuzzy metric spaces such as openness of open balls whereas it is not held in general for b-metric and fuzzy b-metric spaces. The aim of the present paper is to go further in the research of fuzzy sbmetric spaces. Interesting examples are also presented to support our results.

\section{Preliminaries}

In this section, some pertinent concepts are presented from the existing literature. These concepts will be helpful to understand the results which are established in the present research.

Definition 1 (see [9]). Consider $\Omega$ be a nonempty set. A fuzzy set in $\Omega$ is a function with domain $\Omega$ and values in $[0,1]$, i.e., $B$ is a fuzzy set if $B: \Omega \longrightarrow[0,1]$ is a function. If $B$ is a fuzzy set and $a \in \Omega$, then the functional value $B(a)$ is called the grade of membership of $a$ in $B$.

Definition 2 (see [3]). A mapping $G$ from $[0,1] \times[0,1]$ to $[0,1]$ is called continuous triangular norm (t-norm) or a conjunction if it satisfies

(1) Symmetry: $G(a, b)=G(b, a)$, for $a, b \in[0,1]$

(2) Monotonicity: $G\left(a_{1}, b_{1}\right) \leq G\left(a_{2}, b_{2}\right) \quad$ whenever $a_{1} \leq a_{2}$ and $b_{1} \leq b_{2}$

(3) Associativity: $G(G(a, b), c)=G(a, G(b, c))$, where $a, b, c, \in[0,1]$

(4) Boundary condition: $G(1, a)=a$, for all $a \in[0,1]$

The following are three basic t-norms.

Example 1. Three basic t-norms are defined as below:

(1) The minimum triangular norm: $G\left(a_{1}, a_{2}\right)=$ $\min \left(a_{1}, a_{2}\right)$

(2) The product triangular norm: $G\left(a_{1}, a_{2}\right)=a_{1} a_{2}$

(3) The Lukasiewicz triangular norm: $G\left(a_{1}, a_{2}\right)=$ $\max \left(a_{1}+a_{2}-1,0\right)$.

Definition 3 (see [14]). If $\Omega$ is an arbitrary set, $*$ is t-norm and $M$ is a fuzzy set in $\Omega \times \Omega \times[0, \infty)$ such that $\forall a, b, c \in \Omega$; then, triple $(\Omega, M, *)$ is known to be fuzzy metric space if it hold following axioms:

$$
\begin{aligned}
& (M 1) M(a, b, 0)=0 \\
& (M 2) M(a, b, u)=1, \quad \forall u>0 \text { iff } a=b \\
& (M 3) M(a, b, u)=M(b, a, u), \quad \forall u>0
\end{aligned}
$$

$(M 4) M(a, c, u+v) \geq M(a, b, u) * M(b, c, v), \quad \forall a, b, c$ $\in \Omega, \forall u, v \geq 0$

$(M 5) M(a, b,):. \mathbb{R}^{+} \cup\{0\} \longrightarrow[0,1]$ is left continuous

Example 2. Let $M: \Omega \times \Omega \times \mathbb{R}^{+} \cup\{0\} \longrightarrow[0,1]$, and define $M$ by

$$
\begin{aligned}
& M(\eta, \lambda, u)=\frac{\min \{\eta, \gamma\}+u}{\max \{\eta, \gamma\}+u} \\
& \forall \eta, \lambda \in \Omega \text { and } u \geq 0 \text { is a fuzzy metric. }
\end{aligned}
$$

Definition 4 (see [22]). Let $\Omega$ be an arbitrary nonempty set, $s \geq 1$ be arbitrary real number, and $*$ be a t-norm. $M$ is a fuzzy set in $\Omega \times \Omega \times[0, \infty)$; it is known as fuzzy sb-metric if $\forall a, b, c \in \Omega$; the following axioms satisfied

$$
\begin{aligned}
& (s b M 1) M(a, b, 0)=0 \\
& (s b M 2) M(a, b, u)=1 \text { iff } a=b \\
& (s b M 3) M(a, b, u)=M(b, a, u), u \geq 0 \\
& (s b M 4) \\
& M(a, c, u+s . k) \geq M(a, b, u) * M(b, c, k), u, k \geq 0 \\
& \text { (sbM5) } M(a, b, .): \mathbb{R}^{+} \cup\{0\} \longrightarrow[0,1] \quad \text { is left } \\
& \text { continuous }
\end{aligned}
$$

The quadruple $(\Omega, M, *, s)$ is known as fuzzy sb-metric space.

Remark 1. Consider $(\Omega, M, *, s)$ be a fuzzy sb-metric space.

(1) Let $\left(b_{n}\right)$ be a sequence in $\Omega$. $\left(b_{n}\right)$ is said to be convergent and converges to $b \in \Omega$ if $\lim _{n \rightarrow \infty} M\left(b_{n}, b, k\right)=$ 1 for each $k>0$.

(2) The sequence $\left(a_{n}\right)$ is said to be a Cauchy sequence if, for any $0<\epsilon<1$ and for each $u>0$, there exists a natural number $n_{0}$ such that $M\left(a_{n}, a_{m}, u\right)>1-\in$ for all natural numbers $n, m \geq n_{0}$

(3) A fuzzy sb-metric space in which every Cauchy sequence is convergent is called complete.

Definition 5 (see [19]). Let $\Omega=(\Omega, d)$ be a metric space. A mapping $G: \Omega \longrightarrow \Omega$ is known as Banach contraction on $G$ if there is a positive real number $0<\alpha<1$ such that $\forall a, b \in \Omega$ :

$$
d(G a, G b) \leq \alpha d(a, b)
$$

Definition 6 (see [23]). Let $(\Omega, d)$ be a metric space and $G: \Omega \longrightarrow \Omega$ be a mapping if $\exists \alpha \in(0,1 / 2)$ such that, for all $a_{1}, a_{2} \in \Omega$, we have

$$
d\left(G a_{1}, G a_{2}\right) \leq \alpha\left\{d\left(a_{1}, G a_{1}\right)+d\left(a_{2}, G a_{2}\right)\right\} .
$$

Then, $G$ is known as Kannan contraction. 
Definition 7 (see [24]). Let $(\Omega, d)$ be a metric space and $G: \Omega \longrightarrow \Omega$ be a mapping if there exist $\alpha \in(0,1 / 2)$ such that, for all $a_{1}, a_{2} \in \Omega$, we have

$$
d\left(G a_{1}, G a_{2}\right) \leq \alpha\left\{d\left(a_{1}, G a_{2}\right)+d\left(a_{2}, G a_{1}\right)\right\} .
$$

Then, $G$ is known as Chatterjee contraction.

\section{Fixed Points in Fuzzy Sb-Metric Spaces}

In this section, we have established and proved some important results which ensure the existence and uniqueness of fixed points in fuzzy sb-metric spaces for single-valued continuous and discontinuous mappings. Examples are also created to give the strength of these results.

Example 3. Let $(\Omega, d, s)$ be sb-metric space. Let $M: \Omega \times \Omega \times[0, \infty) \longrightarrow[0,1]:$

$$
M\left(\omega_{1}, \omega_{2}, \eta\right)=\left\{\begin{array}{l}
e^{-\left(d\left(\omega_{1}, \omega_{2}\right) / \eta\right)} \quad \text { if } \eta>0 \\
0 \quad \text { if } \eta=0
\end{array}\right.
$$

Then, $(\Omega, M, \wedge, s)$ is fuzzy strong b-metric space, where ${ }^{\wedge}$ is minimum t-norm.

Solution 1. We will just check (sbM4) because the rest are trivial.

For this, let $\omega_{1}, \omega_{2}, \omega_{3} \in \Omega$ and $\eta, k \geq 0$; without restraining the generality, we assume that $M\left(\omega_{1}, \omega_{2}, \eta\right) \leq M\left(\omega_{2}, \omega_{3}, k\right)$. Thus $e^{-\left(d\left(\omega_{1}, \omega_{2}\right) / \eta\right)} \leq e^{-\left(d\left(\omega_{2} \text {, }\right.\right.}$ $\left.\left.\omega_{3}\right) / k\right)$.

This implies $\quad-\left(d\left(\omega_{1}, \omega_{2}\right) / \eta\right) \leq-\left(\left(d \omega_{2}, \omega_{3}\right) / k\right)$, $\Rightarrow d\left(\omega_{2}, \omega_{3}\right) / k \leq d\left(\omega_{1}, \omega_{2}\right) / \eta$, Or $\eta d\left(\omega_{2}, \omega_{3}\right) \leq k d\left(\omega_{1}, \omega_{2}\right)$.

On the contrary,

$$
\begin{aligned}
M\left(\omega_{1}, \omega_{3}, \eta+s . k\right) & =e^{-\left(d\left(\omega_{1}, \omega_{3}\right) / \eta+s . k\right)} \\
& \leq e^{-\left(d\left(\omega_{1}, \omega_{2}\right)+s d\left(\omega_{2}, \omega_{3}\right) / \eta+s . k\right)} .
\end{aligned}
$$

Now, we will show that $e^{-\left(d\left(\omega_{1}, \omega_{2}\right)+s d\left(\omega_{2}, \omega_{3}\right) / \eta+s . k\right)} \geq$ $e^{-\left(d\left(\omega_{1}, \omega_{2}\right) / \eta\right)}$.
Hence, we will obtain that $M\left(\omega_{1}, \omega_{3}, \eta+s . k\right) \geq$ $M\left(\omega_{1}, \omega_{2}, \eta\right)=M\left(\omega_{1}, \omega_{2}, \eta\right) \wedge M\left(\omega_{2}, \omega_{3}, k\right)$.

We remark that

$$
\begin{aligned}
& e^{-\left(d\left(\omega_{1}, \omega_{2}\right)+s d\left(\omega_{2}, \omega_{3}\right) / \eta+s . k\right)} \geq e^{-\left(d\left(\omega_{1}, \omega_{2}\right) / \eta\right),} \\
\Rightarrow & \frac{d\left(\omega_{1}, \omega_{2}\right)}{\eta} \geq \frac{d\left(\omega_{1}, \omega_{2}\right)+s d\left(\omega_{2}, \omega_{3}\right)}{\eta+s \cdot k}, \\
\Leftrightarrow & (\eta+s . k) d\left(\omega_{1}, \omega_{2}\right) \geq \eta\left(d\left(\omega_{1}, \omega_{2}\right)+s d\left(\omega_{2}, \omega_{3}\right)\right), \\
\Leftrightarrow & k d\left(\omega_{1}, \omega_{2}\right) \geq \eta d\left(\omega_{2}, \omega_{3}\right),
\end{aligned}
$$

which is true.

Example 4. Consider $(\Omega, d, t)$ be sb-metric space. Let $\quad M_{d}: \Omega * \Omega *[0, \infty) \longrightarrow[0,1], \quad M_{d}\left(a_{1}, a_{2}, t\right)=$ $\left\{\begin{array}{l}t / t+d\left(a_{1}, a_{2}\right) \quad \text { if } t>0 \\ 0 \quad \text { if } t=0\end{array}\right.$

Then, $\left(\Omega, M_{d}, \wedge, s\right)$ is a fuzzy sb-metric space, where $\wedge$ is minimum t-norm.

Theorem 1. Suppose $(\Omega, M, *, s)$ be a complete fuzzy sbmetric space, * be a continuous t-norm, $M(a, b, u)$ be strictly increasing in variable $u$, and

$$
\lim _{u \longrightarrow \infty} M(a, b, u)=1, \quad \forall a, b \in \Omega .
$$

Let $G: \Omega \longrightarrow \Omega$ be a mapping satisfying $M(G a$, $G b, k u) \geq M(a, b, u)$, for all $a, b \in \Omega$, where $0<k<1$. Then, there exist a unique fixed point of $G$

Proof. Consider $a_{0} \in X$ be any arbitrary element and let $a_{n}$ be a sequence in $\Omega$ so that

$$
\begin{aligned}
a_{n} & =G a_{n-1} \\
& =G^{n} a_{0}(n \in \mathbb{N}) .
\end{aligned}
$$

Now,

$$
\begin{aligned}
M\left(a_{n}, a_{n+1}, k u\right)= & M\left(G^{n} a_{0}, G^{n+1} a_{0}, k u\right) \geq M\left(G^{n-1} a_{0}, G^{n} a_{0}, u\right) \\
= & M\left(a_{n-1}, a_{n}, u\right) \\
= & M\left(G^{n-1} a_{0}, G^{n} a_{0}, u\right) \\
= & M\left(G^{n-1} a_{0}, G^{n} a_{0}, u\right) \geq M\left(G^{n-2} a_{0}, G^{n-1} a_{0}, \frac{u}{k}\right) \\
= & M\left(a_{n-2}, a_{n-1}, u / k\right) \cdots \geq M\left(a_{0}, a_{1}, \frac{u}{k^{n-1}}\right) \\
& \text { So, } M\left(a_{n}, a_{n+1}, k u\right) \geq M\left(a_{0}, a_{1}, \frac{u}{k^{n-1}}\right) .
\end{aligned}
$$

For every $n \in \mathbb{N}$ and $u \geq 0$ and thus for any integer $p>0$, by using ( $s$ bM4), we obtain 


$$
\begin{aligned}
M\left(a_{n}, a_{n+p}, u\right) \geq & M\left(a_{n}, a_{n+1}, \frac{u}{2}\right) * M\left(a_{n+1}, a_{n+p}, \frac{u}{2 s}\right) \\
\geq & M\left(a_{n}, a_{n+1}, \frac{u}{2}\right) * M\left(a_{n+1}, a_{n+2}, \frac{u}{4 s}\right) * M\left(a_{n+2}, a_{n+p}, \frac{u}{4 s^{2}}\right) \\
\geq & M\left(a_{n}, a_{n+1}, \frac{u}{2}\right) * M\left(a_{n+1}, a_{n+2}, \frac{u}{4 s}\right) * M\left(a_{n+2}, a_{n+3}, \frac{u}{8 s^{2}}\right) * M\left(a_{n+3}, a_{n+p}, \frac{u}{8 s^{3}}\right) \\
\geq & M\left(a_{n}, a_{n+1}, \frac{u}{2}\right) * M\left(a_{n+1}, a_{n+2}, \frac{u}{4 s}\right) * M\left(a_{n+2}, a_{n+3}, \frac{u}{8 s^{2}}\right) * M\left(a_{n+3}, a_{n+4}, \frac{u}{8 s^{3}}\right) \\
& * \ldots * M\left(a_{n+p-1}, a_{n+p}, \frac{u}{2^{p^{-1}} s^{p-1}}\right) .
\end{aligned}
$$

Using (10), we have

$$
M\left(a_{n}, a_{n+p}, u\right) \geq M\left(a_{0}, a_{1}, \frac{u}{2 k^{n}}\right) * M\left(a_{0}, a_{1}, \frac{u}{2^{2} k^{n+1}}\right) * \ldots * M\left(a_{0}, a_{1}, \frac{u}{2^{p-1} s^{p-1} k^{n+p-1}}\right)
$$

As $n \longrightarrow \infty k^{n} \longrightarrow 0$, this implies that $u / 2 k^{n} \longrightarrow \infty$, so by using (8), we have $M\left(a_{n}, a_{n+p}, u\right) \geq 1 * 1 * 1 \ldots * 1$ (p times). This implies that $M\left(a_{n}, a_{n+p}, u\right) \geq 1$; this gives that $a_{n}$ is a Cauchy sequence. Given $\Omega$ is complete so, there exist a point $b$ in $\Omega$ such that $\lim a_{n}=b$.

Now, using $(s b M 4)$,

$$
\begin{aligned}
& M(b, G b, u) \geq M\left(b, a_{n+1}, \frac{u}{2}\right) * M\left(a_{n+1}, G b, \frac{u}{2 s}\right) \geq M\left(b, a_{n+1}, \frac{u}{2}\right) * M\left(G a_{n}, G b, \frac{u}{2 s}\right), \\
& M(b, G b, u) \geq M\left(b, a_{n+1}, \frac{u}{2}\right) * M\left(a_{n}, b, \frac{u}{2 s k}\right) .
\end{aligned}
$$

In limiting case, when $n \longrightarrow \infty$, we have

$$
M(b, G b, u) \geq M\left(b, b, \frac{u}{2}\right) * M\left(b, b, \frac{u}{2 s k}\right)=1 * 1,
$$

which implies $M(b, G b, u) \geq 1$. So, $G b=b$.

Uniqueness: let $b, b^{*}$ be two fixed points of mapping $G$; then, $G b=b$ and $G b^{*}=b^{*}$.

$M(G b, b, u)=1$ and $M\left(G b^{*}, b^{*}, u\right)=1$.

Now, $\quad M\left(b, b^{*}, u\right)=M\left(G b, G b^{*}, u\right) \geq M\left(b, b^{*}, u / k\right)$, which is a contradiction to the fact that $M(a, b, u)$ is strictly increasing in variable $u$. So, $b=b^{*}$

Example 5. Let $\Omega=[0,1] \subset R$ and $M: \Omega \times \Omega \times[0, \infty)$ $\longrightarrow[0,1]$ be defined as

$$
M(x, y, \eta)= \begin{cases}\frac{\eta}{\eta+|x-y|} & \text { if } \eta>0 \\ 0 \quad \text { if } \eta=0 . & \end{cases}
$$

Let $G: \Omega \longrightarrow \Omega$ be defined by $G x=x / 6$ and $k=1 / 3$, and * is minimum t-norm.
We have $M(G x, G y, k \eta)=\eta / 3 / \eta / 3+|G x-G y|=\eta / 3 / \eta /$ $3+|x-y / 6|=\eta / \eta+|x-y| / 2 \geq \eta / \eta+|x-y| \geq \eta / \eta+|x-y|$ $=M(x, y, \eta)$.

This implies $M(G x, G y, k \eta) \geq M(x, y, \eta)$. So, $G$ contains a unique fixed point.

Corollary 1. Consider a complete fuzzy metric space $(\Omega, M, *)$, * be a continuous t-norm, and $M(a, b, u)$ is strictly increasing in variable $u$ and $\lim _{u \longrightarrow \infty} M(a, b, u)$ $=1, \quad \forall a, b \in \Omega$.

Let $G: \Omega \longrightarrow \Omega$ be a mapping satisfying $M(G a, G b, k u) \geq M(a, b, u)$, for all $a, b \in \Omega$, where $0<k<1$. Then, there exists a unique fixed point of $G$.

Theorem 2. Let $(\Omega, M, *, s)$ be a complete fuzzy sb-metric space, where $*$ is a continuous t-norm, defined as $*=\min \left\{x_{1}, x_{2}\right\}$, and $M(a, b, u)$ is strictly increasing in variable $u$ and

$$
\lim _{u \longrightarrow \infty} M(a, b, u)=1, \quad \forall a, b \in \Omega .
$$


Let $G: \Omega \longrightarrow \Omega$ be a self-mapping which satisfies the given axioms $\forall a, b \in \Omega$ :

$$
M(G a, G b, k u) \geq M(a, G a, u) * M(b, G b, u),
$$

where $u \geq 0$ and $0<k<1$. Then, there will be a unique fixed point of $G$.

Proof. Consider $a_{o} \in \Omega$; then, $G a_{o} \in \Omega$. Let $a_{1} \in \Omega$ such that $a_{1}=G a_{0}$.
By induction, we find a sequence $a_{n}=G a_{n-1}$, in $\Omega$.

Now, $\quad M\left(a_{n}, a_{n+1}, k u\right)=M\left(G a_{n-1}, G a_{n}, k u\right) \geq M\left(a_{n-1}\right.$, $\left.G a_{n-1}, u\right) * M\left(a_{n}, G a_{n}, u\right) \geq M\left(a_{n-1}, a_{n}, u\right) * M\left(a_{n}, a_{n+1}, u\right)$

Since $M(a, b, u)$ is strictly increasing in variable $u$ and $k u<u$, so, we cannot write

$$
M\left(a_{n}, a_{n+1}, k u\right) \geq M\left(a_{n}, a_{n+1}, u\right) .
$$

Therefore, $=M\left(G a_{n-2}, G a_{n-1}, u\right)$ :

$$
\begin{aligned}
& \geq M\left(a_{n-2}, G a_{n-2}, u / k\right) * M\left(a_{n-1}, G a_{n-1}, u / k\right) \\
& \geq M\left(a_{n-2}, a_{n-1}, u / k\right) * M\left(a_{n-1}, a_{n}, u / k\right) \geq M\left(a_{n-2}, a_{n-1}, u / k\right) \\
\cdots & \geq M\left(a_{0}, a_{1}, \frac{u}{k^{n-1}}\right), \\
M\left(a_{n}, a_{n+1}, k u\right) & \geq M\left(a_{o}, a_{1}, \frac{u}{k^{n-1}}\right) .
\end{aligned}
$$

Now, let $p$ be a positive integer, and using ( $s b M 4)$, we have

$$
\begin{aligned}
M\left(a_{n}, a_{n+p}, u\right) \geq & M\left(a_{n}, a_{n+1}, \frac{u}{2}\right) * M\left(a_{n+1}, a_{n+p}, \frac{u}{2 s}\right) \\
\geq & M\left(a_{n}, a_{n+1}, \frac{u}{2}\right) * M\left(a_{n+1}, a_{n+2}, \frac{u}{4 s}\right) * M\left(a_{n+2}, a_{n+p}, \frac{u}{4 s^{2}}\right) \\
\geq & M\left(a_{n}, a_{n+1}, \frac{u}{2}\right) * M\left(a_{n+1}, a_{n+2}, \frac{u}{4 s}\right) * M\left(a_{n+2}, a_{n+3}, \frac{u}{8 s^{2}}\right) \\
& * M\left(a_{n+3}, a_{n+4}, \frac{u}{8 s^{3}}\right) * \ldots * M\left(a_{n+p-1}, a_{n+p}, \frac{u}{2^{p-1} s^{p-1} k^{n+p-1}}\right) .
\end{aligned}
$$

By using inequality (19), we have

$$
M\left(a_{n}, a_{n+p}, u\right) \geq M\left(a_{o}, a_{1}, \frac{u}{2 k^{n}}\right) * M\left(a_{o}, a_{1}, \frac{u}{2^{2} s k^{n+1}}\right) * \ldots * M\left(a_{o}, a_{1}, \frac{u}{2^{p} s^{p-1} k^{n+p-1}}\right) .
$$

Since $0<k<1$, so when $n \longrightarrow \infty, k^{n} \longrightarrow 0$. So, $\lim M\left(a_{o}, a_{n+p}, u\right) \geq 1 * 1 * 1 * \ldots=1$, which implies $a_{n}$ is $\stackrel{n}{\mathrm{a}} \vec{C}^{\infty}$ auchy sequence in $\Omega$. Given $\Omega$ is complete, so, there exist

$b$ in $\Omega$ such that $\lim _{n \rightarrow \infty} a_{n}=b$. Now, using contractive condition,

$$
M\left(G a_{n}, G b, k u\right) \geq M\left(a_{n}, G a_{n}, u\right) * M(b, G b, u) \geq M\left(a_{n}, a_{n+1}, u\right) * M(b, G b, u)
$$

In a limiting case, $n \longrightarrow \infty$ : 
$M(b, G b, k u) \geq M(b, b, u) * M(b, G b, u)=1 * M(b, G b, u)$,

$M(b, G b, k u) \geq M(b, G b, u)$,

which is a contraction to the supposition that $\mathrm{M}(\mathrm{a}, b, \mathrm{u})$ is strictly increasing in variable $u$.

Hence, $G b=b$. So, $b$ is a fixed point of $G$.

Uniqueness: consider two fixed point $b$ and $b^{*}$ of $G$. So, $G b=b$ and $G b^{*}=b^{*}$.

Now, $\quad M\left(b, b^{*}, u\right)=M\left(G b, G b^{*}, u\right) \geq M(b, G b, u / k)$ * $M\left(b^{*}, G b^{*}, u / k\right) \geq 1 * 1$ :

$$
M\left(b, b^{*}, u\right) \geq 1 \Rightarrow b=b^{*} .
$$

Example 6. Let $\Omega=[0,1] \subset R$ and $G: \Omega \times \Omega \times[0, \infty) \longrightarrow$ $[0,1]$ be defined as

$$
M(x, y, \eta)=\left\{\begin{array}{l}
\frac{\eta}{\eta+|x-y|} \quad \text { if } \eta>0, \\
0 \quad \text { if } \eta=0 .
\end{array}\right.
$$

Let $G: \Omega \longrightarrow \Omega$ be defined by $G x=x / 30$ and $k=2 / 3$, and $*$ is minimum $\mathrm{t}$-norm. Without losing generality, we let $x>y$ and $M(x, G x, \eta) * M(y, G y, \eta)=M(x, G x, \eta)$. Then, we have to prove that $M(G x, G x, k \eta) \geq M(x, G x, \eta)$.

Now, as $x, y \in[0,1]$, we have $|x-y / 30| \leq|x+y / 30|=$ $1 / 3|3 x / 30+3 y / 30| \leq 1 / 3|29 x / 30+29 y / 30| \leq 1 / 3(|29 x / 30|$ $+|29 y / 30|)$.

This implies

$3\left|\frac{x-y}{30}\right| \leq\left|\frac{29 x}{30}\right|+\left|\frac{29 y}{30}\right| \Leftrightarrow(2) \frac{3}{2}\left|\frac{x-y}{30}\right| \leq\left|\frac{29 x}{30}\right|+\left|\frac{29 y}{30}\right|$.

Using result of analysis, if $a, b, c>0$ and $2 a \leq b+c$, then $a \leq \max \{b, c\}$.

So, $1 / 3|x-y / 30| \leq|29 x / 30|$. As $t>0$, we can write as $\eta+$ $3 / 2|x-y / 30| \leq \eta+|29 x| \quad 30|\Rightarrow \eta / \eta+3 / \quad 2| x-y / 30 \mid \leq \eta / \eta+$ $|x-y / 30| \Leftrightarrow M(G x, G y, 2 / 3 \eta) \geq M(x, G x, \eta)=M(x, G x, \eta)$ * $M(y, G y, \eta)$. $\Omega$.

So, by the above theorem, $G$ has a unique fixed point in

Corollary 2. Consider a complete fuzzy metric space $(\Omega, M, *)$, where $*$ is a continuous t-norm, given by $x_{1} * x_{2}=\min \left\{x_{1}, x_{2}\right\}$ and $M(a, b, u)$ is strictly increasing in variable $u$ and

$$
\lim _{u \longrightarrow \infty} M(a, b, u)=1, \quad \forall a, b \in \Omega .
$$

Let $G: \Omega \longrightarrow \Omega$ be a self-mapping which satisfies the given condition $\forall a, b \in \Omega$ :

$$
M(G a, G b, k u) \geq M(a, G a, u) * M(b, G b, u),
$$

where $u \geq 0$ and $0<k<1$. Then, there will be a unique fixed point of $G$.

Theorem 3. Consider a complete fuzzy metric space $(\Omega, M, *, s)$, where $*$ is a $t$-norm, given by $*=\min \left\{x_{1}, x_{2}\right\}$ and $M(a, b, u)$ is strictly increasing in variable $u$ and $\lim M(a, b, u)=1, \quad \forall a, b \in \Omega$. Let $G: \Omega \longrightarrow \Omega$ be a self$\stackrel{u \rightarrow}{\rightarrow a p p i n g}$, satisfying the given condition $\forall a, b \in \Omega$ :

$$
M(G a, G b, k u) \geq M(a, G b, u) * M(b, G a, u)
$$

where $u \geq 0$ and $0<k<1 / 2 s$. Then, there exists a unique fixed point of $G$.

Proof. Consider $a_{o} \in \Omega$; then, $G a_{o} \in \Omega$. Let $a_{1} \in \Omega$ such that $a_{1}=G a_{0}$.

By induction, we find a sequence $a_{n}=G a_{n-1}$. $M(x, G x, \eta) * M(y, G y, \eta)$ in $\Omega$.

Now, $\quad M\left(a_{n}, a_{n+1}, k u\right)=M\left(G a_{n-1}, G a_{n}, k u\right) \geq M\left(a_{n-1}\right.$, $\left.G a_{n}, u\right) * M\left(a_{n}, G a_{n-1}, u\right) \geq M\left(a_{n-1}, a_{n+1}, u\right) * M\left(a_{n}, a_{n}, u\right)$.

Since $M\left(a_{n}, a_{n}, u\right)=1$, so, $M\left(a_{n}, a_{n+1}, k u\right) \geq M\left(a_{n-1}\right.$, $\left.a_{n+1}, u\right)$.

By using triangular inequality of fuzzy sb-metric space, we have

$$
M\left(a_{n}, a_{n+1}, k u\right) \geq M\left(a_{n-1}, a_{n}, \frac{u}{2}\right) * M\left(a_{n}, a_{n+1}, \frac{u}{2 s}\right) .
$$

Since $M(a, b,$.$) is strictly increasing in variable u$ and $k u<u / 2 s$, so, we cannot write

$$
M\left(a_{n}, a_{n+1}, k u\right) \geq M\left(a_{n}, a_{n+1}, \frac{u}{2 s}\right) .
$$

Therefore,

$$
\begin{aligned}
M\left(a_{n}, a_{n+1}, k u\right) & \geq M\left(a_{n-1}, a_{n}, \frac{u}{2}\right)=M\left(G a_{n-2}, G a_{n-1}, \frac{u}{2}\right) \\
& \geq M\left(a_{n-2}, G a_{n-1}, \frac{u}{2 k}\right) * M\left(a_{n-1}, G a_{n-2}, \frac{u}{2 k}\right) \\
& =M\left(a_{n-2}, a_{n}, \frac{u}{2 k}\right) * M\left(a_{n-1}, a_{n-1}, \frac{u}{2 k}\right) .
\end{aligned}
$$

Since $M\left(a_{n-1}, a_{n-1}, u / 2 k\right)=1$, so, $M\left(a_{n}, a_{n+1}, k u\right) \geq$ $M\left(a_{n-2}, a_{n}, u / 2 k\right)$.

Using ( $s b M 4)$, we obtain

$$
M\left(a_{n}, a_{n+1}, k u\right) \geq M\left(a_{n-2}, a_{n-1}, \frac{u}{4 k}\right) * M\left(a_{n-1}, a_{n}, \frac{u}{4 s k}\right) .
$$

As $M(a, b,$.$) is an increasing function in variable u$, so, we can only write

$$
\begin{aligned}
M\left(a_{n}, a_{n+1}, k u\right) & \geq M\left(a_{n-2}, a_{n-1}, \frac{u}{4 k}\right) \\
& =M\left(G a_{n-3}, G a_{n-2}, \frac{u}{4 k}\right) .
\end{aligned}
$$


Using (29), we can write

$$
\begin{aligned}
M\left(a_{n}, a_{n+1}, k u\right) & \geq M\left(a_{n-2}, G a_{n-3}, \frac{u}{4 k^{2}}\right) * M\left(a_{n-3}, G a_{n-2}, \frac{u}{4 k^{2}}\right) \\
& \geq M\left(a_{n-2}, a_{n-2}, \frac{u}{4 k^{2}}\right) * M\left(a_{n-3}, a_{n-1}, \frac{u}{4 k^{2}}\right)=1 * M\left(a_{n-3}, a_{n-1}, \frac{u}{4 k^{2}}\right) .
\end{aligned}
$$

So, $M\left(a_{n}, a_{n+1}, k u\right) \geq M\left(a_{n-3}, a_{n-1}, u / 4 k^{2}\right)$.

Using ( $s b M 4)$, we can write

$$
M\left(a_{n}, a_{n+1}, k u\right) \geq M \geq\left(a_{n-3}, a_{n-2}, \frac{u}{8 k^{2}}\right) * M\left(a_{n-2}, a_{n-1}, \frac{u}{8 s k^{2}}\right) .
$$
$\left.u / 8 k^{2}\right)$.

Only possibility is $M\left(a_{n}, a_{n+1}, k u\right) \geq M\left(a_{n-3}, a_{n-2}\right.$,

Continuing this process, we have

$$
M\left(a_{n}, a_{n+1}, k u\right) \geq M\left(a_{o}, a_{1}, \frac{u}{2^{n} k^{n-1}}\right) .
$$

$$
\begin{aligned}
M\left(a_{n}, a_{n+p}, u\right) \geq & M\left(a_{n}, a_{n+1}, \frac{u}{2}\right) * M\left(a_{n+1}, a_{n+p}, \frac{u}{2 s}\right) \\
\geq & M\left(a_{n}, a_{n+1}, \frac{u}{2}\right) * M\left(a_{n+1}, a_{n+2}, \frac{u}{4 s}\right) * M\left(a_{n+2}, a_{n+p}, \frac{u}{4 s^{2}}\right) \\
\geq & M\left(a_{n}, a_{n+1}, \frac{u}{2}\right) * M\left(a_{n+1}, a_{n+2}, \frac{u}{4 s}\right) * \\
& M\left(a_{n+2}, a_{n+3}, \frac{u}{8 s^{2}}\right) * M\left(a_{n+3}, a_{n+4}, \frac{u}{8 s^{3}}\right) * \cdots * M\left(a_{n+p-1}, a_{n+p}, \frac{u}{2^{p} s^{p-1}}\right) .
\end{aligned}
$$

Using (37), we can write

$$
M\left(a_{n}, a_{n+p}, u\right) \geq M\left(a_{o}, a_{1}, \frac{u}{2^{n+1} k^{n}}\right) * M\left(a_{0}, a_{1}, \frac{u}{2^{n+2} s k^{n+1}}\right) * \cdots * M\left(a_{o}, a_{1}, \frac{u}{2^{n+p} s^{p-1} k^{n+p-1}}\right) .
$$

Since $0<k<1$ and $n \longrightarrow \infty$ implies that $u / 2^{n+1} k^{n} \longrightarrow \infty$, so, $\lim M\left(a_{n}, a_{n+p}, u\right) \geq 1 * 1 * \cdots *$ $1=1$, which implies $a_{n}$ is a Cauchy sequence in $\Omega$. Given $\Omega$ is complete, so there exist $b \in \Omega$ such that $\lim a_{n}=b$.

Now, by using contractive condition,

$$
\begin{aligned}
M\left(G a_{n}, G b, k u\right) & \geq M\left(b, G a_{n}, u\right) * M\left(a_{n}, G b, u\right) \\
& \geq M\left(b, a_{n+1}, u\right) * M\left(a_{n}, G b, u\right) .
\end{aligned}
$$

In a limiting case, as $n \longrightarrow \infty$, $M(b, G b, k u) \geq M(b, b, u) * M(b, G b, u)=1 * M(b, G b, u)$. $b=G b$.

$M(b, G b, k u) \geq M(b, G b, u)$ which is a contradiction, so,

Uniqueness: let $b$ and $b^{*}$ be two fixed points of $G$. $M(b, G b, u)=1$ and $M\left(b^{*}, G b^{*}, u\right)=1$. 
Now, $\quad M\left(b, b^{*}, u\right)=M\left(G b, G b^{*}, u\right) \geq M\left(b, G b^{*}, u / k\right)$ $* M\left(b^{*}, G b, u / k\right) \geq M\left(b, b^{*}, u / k\right) * M\left(b^{*}, b, u / k\right)$, which implies that $M\left(b, b^{*}, u\right) \geq M\left(b, b^{*}, u / k\right)$, which contradicts the fact that $M(a, b,$.$) is strictly increasing in variable. So,$ $b=b^{*}$.

Corollary 3. Consider a complete fuzzy metric $(\Omega, M, *)$, where $*$ is a continuous t-norm, given by $x_{1} * x_{2}=$ $\min \left\{x_{1}, x_{2}\right\}$, and $M(a, b, u)$ is strictly increasing in variable $u$ and

$$
\lim _{u \longrightarrow \infty} M(a, b, u)=1, \quad \forall a, b \in \Omega .
$$

Let $G: \Omega \longrightarrow \Omega$ be a self-mapping which satisfies the given condition, for all $a, b \in \Omega$,

$$
M(G a, G b, k u) \geq M(a, G b, u) * M(b, G a, u),
$$

where $u \geq 0$ and $0<k<1 / 2$. Then, $\exists$ is a unique fixed point of G.

\section{Conclusion}

Fixed-point techniques are very useful and attractive tools for researchers. This theory has potential applications in functional inclusions, optimization theory, fractal graphics, discrete dynamics for set-valued operators, and other areas of nonlinear functional analysis. Integral equations arise in several problems in mathematical physics, control theory, critical point theory for nonsmooth energy functionals, differentials, variational inequalities, fuzzy set arithmetic, traffc theory, etc. These can be solved by fixed-point methods.

Fuzzy strong b-metrics, called here by fuzzy sb-metrics, were introduced recently as a fuzzy version of strong b-metrics. It was shown that open balls in fuzzy sb-metric spaces are open in the induced topology (as different from the case of fuzzy b-metric spaces), and thanks to this fact, fuzzy sb-metrics have many useful properties common with fuzzy metric spaces which generally may fail to be in the case of fuzzy b-metric spaces. It is also shown that the class of fuzzy sb-metric spaces lies strictly between the classes of fuzzy metric and fuzzy b-metric spaces. Concerning the further development of the research in the area of fuzzy sb-metrics, we have vision of obtaining some valuable fixed points of some contractive type mappings such as Banach, Kannan, and Chatterjea in these spaces and obtain some corollaries. This work will help researchers in finding the solutions of various type of equations and inequalities. Moreover, our work will motivate researchers to go ahead and establish common fixed points and coincidence points in these spaces for two or more mappings having contractive-type conditions in future.

\section{Data Availability}

The data used to support the finding of the study are included within the article.

\section{Conflicts of Interest}

The authors declare the there are no conflicts of interest regarding the publications.

\section{Acknowledgments}

This work was funded by Deanship of Scientific Research (DSR) King Abdulaziz University, Jeddah. The authors, therefore, acknowledge with thanks DSR for technical and financial support.

\section{References}

[1] A. Azam, "Fuzzy fixed points of fuzzy mappings via a rational inequality," Hacettepe Journal of Mathematics and Statistics, vol. 40, pp. 421-431, 2011.

[2] A. Azam and I. Beg, "Common fixed points of fuzzy maps," Mathematical and Computer Modelling, vol. 49, no. 7-8, pp. 1331-1336, 2009.

[3] A. Azam and S. Kanwal, "Common fixed point results for multivalued mappings in Hausdorff intuitionistic fuzzy metric spaces," Communications in Mathematics and Applications, vol. 9, no. 1, pp. 63-75, 2018.

[4] T. Kamran, M. Samreen, and Q. U. L. Ain, "A generalization of b-metric space and some fixed point theorems," Mathematics, vol. 5, no. 2, 2017.

[5] S. Kanwal and A. Azam, "Common fixed points of intuitionistic fuzzy maps for Meir-keeler type contractions," Advances in fuzzy systems, Article ID 1989423, 6 pages, 2018.

[6] L. Zadeh, "Fuzzy sets," Information and Control, vol. 8, pp. 338-353, 1965.

[7] S. Kanwal and A. Azam, "Bounded lattice fuzzy coincidence theorems with applications," Journal of Inteligence and fuzzy systems, vol. 36, pp. 1-15, 2019.

[8] T. Suzuki, "Mizoguchi-Takahashi's fixed point theorem is a real generalization of Nadler's," Journal of Mathematical Analysis and Applications, vol. 340, no. 1, pp. 752-755, 2008.

[9] D. Wardowski, "Fixed points of a new type of contractive mappings in complete metric spaces," Fixed Point Theory and Applications, vol. 2012, no. 1, p. 94, 2012.

[10] S. Bhunia, G. Ghorai, M. A. Kutbi, M. Gulzar, and M. A. Alam, "On the algebraic characteristics of fuzzy sub e-groups," Journal of Function Spaces, Article ID 5253346, 7 pages, 2021.

[11] M. Gulzar, F. Dilawar, M. H. Mateen, and M. Premkumar, "On some characterization of Q-complex fuzzy sub-rings," The Journal of Mathematics and Computer Science, vol. 22, no. 2021, pp. 295-305, 2021.

[12] M. Gulzar, M. H. Mateen, Y.-M. Chu, D. Alghazzawi, and G. Abbas, "Generalized direct product of complex intuitionistic fuzzy subrings," International Journal of Computational Intelligence Systems, vol. 14, no. 1, pp. 582-593, 2021.

[13] M. Gulzar, F. Dilawar, D. Alghazzawi, and M. H. Mateen, "A note on complex fuzzy subfield," Indonesian Journal of Electrical Engineering and Computer Science, vol. 21, no. 2, pp. 1048-1056, 2021.

[14] I. Kramosil and J. Michálek, "Fuzzy metrics and statistical metric spaces," Kybernetika, vol. 11, no. 5, pp. 336-344, 1975.

[15] A. George and P. Veeramani, "On some results in fuzzy metric spaces,” Fuzzy Sets and Systems, vol. 64, no. 3, pp. 395-399, 1994.

[16] M. Grabiec, "Fixed points in fuzzy metric spaces," Fuzzy Sets and Systems, vol. 27, no. 3, pp. 385-389, 1988. 
[17] J.-x. Fang, "On fixed point theorems in fuzzy metric spaces," Fuzzy Sets and Systems, vol. 46, no. 1, pp. 107-113, 1992.

[18] I. Bakhtin, "The contraction mapping principle in quasimetric spaces," Functional Analysis, vol. 30, pp. 26-37, 1989.

[19] S. Banach, "Sur les opérations dans les ensembles abstraits et leur application aux équations intégrales," Fundamenta Mathematicae, vol. 3, no. 1, pp. 133-181, 1922.

[20] S. Czerwik, "Contraction mappings in b-metric spaces," Acta Mathematica et Informatica Universitatis Ostraviensis, vol. 1, no. 1 , pp. 5-11, 1993.

[21] W. Kirk and N. Shahzad, Fixed Point Theory in Distance Spaces, Springer International Publishing, Manhattan, NY, USA, 2014.

[22] T. Öner and A. Šostak, "Some remarks on fuzzy sb-Metric spaces," Mathematics, vol. 8, no. 12, p. 2123, 2020.

[23] R. Kannan, "Some results on fixed points," Bulletin of the Calcutta Mathematical Society, vol. 60, pp. 71-76, 1968.

[24] S. K. Chatterjea, "Fixed-point theorems," Dokladi na Bolgarskatan Akademiya na Naukite, vol. 25, no. 6, pp. 727-730, 1972. 